\section{CPS-149 INITIAL THERAPY FOR NEOVASCULAR AGE RELATED MACULAR DEGENERATION: ARE THE GUIDELINES MET IN CLINICAL PRACTICE?}

'M Hernando Verdugo*, 'E Queipo García, 'A Pariente Junquera, ${ }^{2}$ Ml López Gálvez, ${ }^{1}$ MT Sánchez Sánchez. ${ }^{1}$ Hospital Clinico Universitario De Valladolid, Pharmacy, Valladolid, Spain; ${ }^{2}$ Hospital Clinico Universitario De Valladolid, Ophthalmology, Valladolid, Spain

\subsection{6/ejhpharm-2020-eahpconf.250}

Background and importance The current clinical practice guidelines for the treatment of neovascular age related macular degeneration (nAMD) consist of a loading phase of 3 monthly intravitreal injections of antivascular endothelial growth factor (anti-VEGF) drugs, followed by individual maintenance pattern. ${ }^{1}$ The treatment of choice is ranibizumab. The response to treatment is conditioned by the time elapsed between diagnosis and initial treatment. ${ }^{2}$

Aim and objectives To analyse the time elapsed between diagnosis and initial treatment in patients with nAMD and to assess compliance with the loading phase.

Material and methods This was an observational retrospective study in patients diagnosed with nAMD who began treatment with anti-VEGF drugs in 2018. Data collected were age, sex, affected eye, neovascular membrane, best corrected visual acuity (BCVA), drug, date of diagnosis and dates of administration of three loading doses. Patients treated bilaterally were counted as two different treatments.

Results Eighty patients were included (61.3\% women, 38.7\% men) with a mean age of $80.3 \pm 8.1$ years. Eighty-three eyes were treated: $48.2 \%(40 / 83)$ right eye and 51.8\% (43/83) left eye, and $84.3 \%(70 / 83)$ received ranibizumab, $12.0 \%$ $(10 / 83)$ bevacizumab and $3.7 \%$ (3/83) aflibercept. Location of the neovascular membrane was subfoveal in $53.0 \%(44 / 83)$, juxtafoveal in $31.3 \%(26 / 83)$ and undefined/unknown in $15.7 \%(13 / 83)$.

Mean BCVA in the right and left eyes were $0.9 \pm 0.8$ logMAR and $0.8 \pm 0.6 \log$ MAR, respectively. Median number of days between diagnosis and first dose was 17 days (0-59), 32 days (18-193) between the first and second doses and 32 days $(18-130)$ between the second and third doses.

Conclusion and relevance

- There was a delay between diagnosis and initial treatment of about 2 weeks, similar to that observed in other studies. ${ }^{2}$ It would be necessary to reduce this time to achieve better vision outcomes.

- The time interval between the three loading doses was considered acceptable. It is important to meet this initial treatment regimen to obtain good results in terms of visual acuity. ${ }^{1}$ It would be interesting to evaluate the real clinical benefit in these patients.

\section{REFERENCES AND/OR ACKNOWLEDGEMENTS}

1. Mitchell $P$, Korobelnik JF, Lanzetta $P$, et al. Ranibizumab (Lucentis) in neovascular age-related macular degeneration: evidence from clinical trials. $\mathrm{Br} J$ Ophthalmol 2010:94:2-13.

2. Abreu-González R, Alberto-Pestano M, Rubio-Rodríguez G, Abreu Reyes P. Delay in age-related macular degeneration treatment in Spain. Arch Soc Esp Oftalmol 2017;92:e31.

No conflict of interest.

\section{CPS-150 ASSESSMENT OF BURDEN OF DISEASE IN TERMS OF HEALTH RELATED QUALITY OF LIFE IN PATIENTS WITH MULTIPLE MYELOMA NOT ELIGIBLE FOR AUTOLOGOUS STEM CELL TRANSPLANTATION}

${ }^{1} O$ Delgado Sanchez ${ }^{*},{ }^{2} \mathrm{~A}$ Domingo, ${ }^{3} \mathrm{~J}$ De La Rubia, ${ }^{4} \mathrm{~A}$ García, ${ }^{5} \mathrm{~A}$ López, ${ }^{6} \mathrm{C}$ Seguí, ${ }^{7} \mathrm{~L}$ Rumi, ${ }^{8} \mathrm{~A}$ Mengual, ${ }^{9} \mathrm{G}$ Lostaunau, ${ }^{10} \mathrm{MV}$ Mateos. ${ }^{1}$ Hospital Son Espases, Pharmacy Department, Palma De Mallorca, Spain; ${ }^{2}$ Hospital General De Granollers, Haematology, Granollers, Spain; ${ }^{3}$ Hospital Dr Peset, Haematology, Valencia, Spain; ${ }^{4}$ Hospital Arnau De Vilanova De Lleida, Haematology, Lleida, Spain; ${ }^{5}$ Hospital Arnau De Vilanova De Valencia, Haematology, Valencia, Spain; ${ }^{6}$ Hospital General De Granollers, Pharmacy Department, Granollers, Spain; ${ }^{7}$ Hospital Arnau De Vilanova De Lleida, Clinical Trials Unit, Lleida, Spain ${ }^{8}$ Hospital Arnau De Vilanova De Valencia, Pharmacy Department, Valencia, Spain; ${ }^{9}$ Celgene Slu, Medical Affairs, Madrid, Spain; ${ }^{10}$ Hospital Universitario Salamanca, Haematology, Salamanca, Spain

\subsection{6/ejhpharm-2020-eahpconf.251}

Background and importance Multiple myeloma (MM) is an incurable disease that is associated with severe symptoms affecting health related quality of life (HRQoL). Here we report the impact of treatment on disease burden in terms of HRQoL and direct health costs.

Aim and objectives Primary endpoints were HRQoL (baseline and at 5 months), reported by EuroQol-5D (EQ-5D) and QLQ (quality of life questionnaire)-C30/QLQ-MY20 questionnaires and direct health costs.

Material and methods QoLMMBuS (NCT 02946333) is an ongoing, prospective, observational study conducted in 53 Spanish sites in patients with MM not eligible for autologous stem cell transplantation (ASCT). The interim cut-off date was November 2018.

Results A total of 161 evaluable patients were enrolled between November 2016 and October 2018. Median age (range) was 77.6 (73.9-82.6) years, $44.7 \%$ were men, and $57.8 \%$ of patients had Eastern Cooperative Oncology Group (ECOG) stage 0/I, 41.6\% stage II (R-ISS), 66.2\% disease isotype $\operatorname{IgG}$ and $33.1 \%$ disease isotype IgA. High risk cytogenetics were detected in $18 \%$ of patients. In total, 156 patients received lenalidomide $(32.0 \%)$ or bortezomib $(68.0 \%)$ as firstline therapy. With a median follow-up of 5.0 months (3.511.1 , response rates were: complete response in $17.9 \%$, very good partial response in $28.4 \%$, partial response in $38.8 \%$ and progression of disease in 1.5\%: $61.5 \%$ of patients remained on firstline therapy. EQ-5D and QLQ C30 (at 5 months) showed an increase in HRQoL mean values for the key domains of global health status/QoL, physical, role and emotional functioning, although patients experienced worsening in dyspnoea $(p=0.003)$. The mean QLQ-MY20 values showed a significant improvement for the domains of disease symptoms $(p=0.037)$ and future perspective $(p=0.010)$ and worsening for side effects and body image. A total of $56.4 \%$ patients experienced at least one adverse event and $77.8 \%$ and $79.9 \%$ of patients went to visit their doctor or outpatients, respectively. Hospital admissions reported were 154 with a mean (SD) time of 18.3 days for hospitalised patients. Mean direct cost of hospitalisation/patient was $6670.9 €$. Annual mean cost was $13748 €$ per patient and $48.5 \%$ of the cost was related to hospitalisation.

Conclusion and relevance Lenalidomide and bortezomib were the main drugs used as firstline treatment of MM patients not 